\title{
Retratamento endodôntico em dente anterior acometido por reabsorção radicular interna: relato de caso
}

\author{
Endodontical retroaction in previous tooth subjected by internal radicular reabsoration: \\ case report
}

\author{
Retratamiento endodóntico en dente anterior acomiendo por reabsoración radicular \\ interna: relato de caso
}

Francyne Aparecida Leão Camêlo¹, Manoelyne Souza Silva1', Nathalia Maria da Rocha Argolo1, Lara Laís de Lima Monezi ${ }^{1 *}$, Dario Fernandes Lopes Neto², Adriana Pachêco de Oliveira ${ }^{3}$.

\section{RESUMO}

Introdução: A reabsorção radicular interna, ou chamada de reabsorção intracanal, odontoblastoma, e granuloma interno, representa um processo patológico de rara ocorrência. Uma vez que a terapêutica da reabsorção interna e externa é diferente, é de suma importância a realização de um diagnóstico diferencial cuidadoso, o qual se baseia principalmente na interpretação radiográfica, sendo complementada pela história do paciente e pelo exame clínico. Discussão: Atualmente, a Tomografia Computadorizada de Feixe Cônico constitui uma ferramenta muito importante, pois atribui maior confiabilidade ao diagnóstico, classificação e planejamento do tratamento. Relato de Caso: O presente trabalho objetivo descrever um caso clínico de retratamento endodôntico acompanhado do reparo de uma reabsorção radicular interna com o uso do cimento reparador biocerâmico, que proporcionou o retorno funcional do dente envolvido, a fim de abordar os aspectos relevantes ao diagnóstico e tratamento.

Palavras-chave: Endodontia, Reabsorção Interna, Cimento Reparador.

\begin{abstract}
Introduction: Internal root resorption, or intracanal reabsorption, odontoblastoma, and internal granuloma, represents a rare pathological process. Since internal and external resorption therapy is different, a careful differential diagnosis, which is mainly based on radiographic interpretation, is of utmost importance and is complemented by the patient's history and clinical examination. Discussion: Cone-Beam Computed Tomography is a very important tool, since it gives greater reliability to the diagnosis, classification and treatment planning. Case Report: The objective of this study was to describe a clinical case of endodontic retreatment accompanied by the repair of an internal root resorption with the use of bioceramic restorative cement, which provided the functional return of the involved tooth in order to address the relevant aspects of the diagnosis and treatment.
\end{abstract}

Key words: Endodontics, Internal Resorption, Repair Cement.

\section{RESUMEN}

Introducción: raíz interna de resorción, or intracanal reabsorción, odontoblastoma, y interno granuloma, representa a rare pathological process. Por lo que se refiere a la evaluación de la calidad de los alimentos, se debe tener en cuenta que, Discusión: Cone-Beam Computed Tomografía es una herramienta muy importante, ya que proporciona mayor seguridad para el diagnóstico, clasificación y tratamiento de la planificación. Caso de estudio: El objetivo de este estudio fue describir el caso clínico del endodontic retreatment de la reparación de una raíz interna de la restauración de la bioceramic restauración de cemento, que proporcionó el retorno funcional de la sustancia necesaria para los aspectos relacionados del diagnóstico y el tratamiento.

Key words: Endodontics, Internal Resorption, Reparación Cement.

${ }^{1}$ Centro Universitário Cesmac - Maceió - Alagoas. * E-mail: monezi 10@hotmail.com

SUBMETIDO EM: 10/2018 


\section{INTRODUÇÃO}

A endodontia é a ciência e arte que envolve a etiologia, a prevenção, o diagnóstico e o tratamento das alterações patológicas da polpa dentária e de suas repercussões na região periapical e consequentemente no organismo. Dentre a gama de patologias que envolvem a polpa e os tecidos de sustentação, um dos quadros mais complexos em termos de tratamento e prognóstico, envolve as reabsorções radiculares (LEONARDO MR e LEAL JM, 2011).

As reabsorções radiculares possuem diferentes classificações, de acordo com seu local de origem, sua natureza e seu padrão de evolução clínica. Desenvolvem-se a partir de um fator desencadeante e na presença de um fator de manutenção (MINUZZI ED, 2017). Em relação ao seu padrão de evolução clínica pode-se considerar que há casos onde a reabsorção é auto limitante, sendo chamados de transitórios, e há aqueles onde a reabsorção é progressiva (NEVILLE BW et al, 2009).

A etiologia das reabsorções radiculares se dá a partir do dano ou alteração química do tecido protetor que reveste a superfície radicular interna. Este dano ou alteração decorre principalmente a partir de um trauma, patologias ou ainda, após procedimentos odontológicos. Quanto ao seu local de origem, as reabsorções radiculares são classificadas como interna e externa e quanto à sua natureza podem ser de origem inflamatória ou reabsorção por substituição (NASCIMENTO GJF et al., 2006).

A reabsorção radicular interna, ou chamada de reabsorção intracanal, odontoblastoma, e granuloma interno, representa um processo patológico de rara ocorrência. (NEVILLE BW et al, 2009). Constituem situações clínicas rara na dentição permanentes e são caracterizadas por um alargamento que fica localizado no espaço pulpar, de forma oval. A reabsorção radicular externa, que é muito mais frequente, é com frequência confundida com a interna. Para evitar erros junto ao tratamento, é de extrema importância que se realize um diagnóstico diferencial de forma correta (FERREIRA MM et al., 2007).

Uma vez que a terapêutica da reabsorção interna e externa é diferente, é de suma importância a realização de um diagnóstico diferencial cuidadoso, o qual se baseia principalmente na interpretação radiográfica, sendo complementada pela história do paciente e pelo exame clínico (BRAMANT CM, BERBET A, 2002). Radiograficamente, a reabsorção radicular interna apresenta-se como uma área radiolúcida, simétrica, com margens nítidas, regulares e bem definidas, de formato arredondado ou oval, variando no tamanho e na localização (SOUZA NETO MD et al., 2001). O tratamento geralmente é a terapia endodôntica e alguns autores acreditam que, quanto mais precoce for à detecção da reabsorção, melhor será o prognóstico em relação ao dente envolvido (CAMARGO SEA, 2008).

A Tomografia Computadorizada por Feixe Cônico (TCFC), também conhecida como Cone Beam, é uma ferramenta muito importante disponível na atualidade, pois possibilita a obtenção de imagens tridimensionais, o que atribui maior confiabilidade aos diagnósticos, classificação e planejamento do tratamento a ser realizado (MENDONÇA TA et al., 2012).

As propriedades do exame tomográfico incluem o seccionamento de imagem 3D em partes mínimas que possibilitam na endodontia: obtenção de diagnóstico das alterações com exatas extensões dos tecidos duros dos dentes e das estruturas perirradiculares, determinação com precisão do número, localização, forma, tamanho e direção das raízes, perfurações radiculares, instrumentos fraturados, obturações retrógradas, reabsorções radiculares, e ainda lesões endo-periodontais (LIMA SMF, REZENDE TMB, 2013).

Os biocerâmicos hoje no âmbito da saúde oral, mais precisamente na endodontia estão representados através dos materiais reparadores. O fato de ter interação e resposta ao estímulo de tecidos vivos alcançou relevância para serem estudados como uma alternativa ao MTA® (Kakani et al., 2015; Debelian e Trope, 2016; Jitaru et al., 2016). A empresa norte americana Brasseler USA® lançou no mercado uma linha de produtos reparadores biocerâmicos com uma apresentação já pré-misturada, tempo de presa reduzido, tolerância na presença de fluídos e com propriedades biológicas semelhantes ao agregado trióxido mineral (AZEVEDO KESQ, 2017). 
A partir de então, novas propostas comerciais de materiais reparadores biocerâmicos têm sido lançadas no mercado odontológico, razão pela qual um grande número de pesquisas e resultados clínicos tem sido divulgado na literatura. Recentemente, um novo material, MTA REPAIR HP — "High Plasticity" MTA (Angelus $\AA^{\circledR}$, Brasil) foi introduzido com a intenção de melhorar algumas das características do MTA original. Esta nova formulação mantém todas as propriedades químicas e biológicas do mesmo, porém com diferentes propriedades físicas de manipulação, resultando em uma maior plasticidade, o que favorece a sua manipulação e inserção. Ademais, sua fórmula utiliza como radiopacificador o tungstato de cálcio em vez de óxido de bismuto, que segundo o fabricante, não provoca a descoloração da raiz ou coroa dentária (ZUOLO ML, 2016).

Desta forma, o presente trabalho objetivou descrever um caso clínico de retratamento endodôntico acompanhado do reparo de uma reabsorção radicular interna com o uso de um cimento reparador de alta plasticidade, a fim de abordar os aspectos relevantes ao diagnóstico e tratamento.

\section{RELATO DE CASO}

Paciente C.S.S., 26 anos, gênero feminino, compareceu para atendimento clínico com a queixa principal referente ao dente 11. O mesmo fora tratado endodonticamente, porém evoluiu com quadro doloroso e com histórico de sangramento excessivo durante as sessões de tratamento, que resultou em manchamento da coroa dentária.

$\mathrm{Na}$ anamnese não houve relato de nenhuma alteração do quadro de saúde, porém, na história odontológica, a paciente referiu hábito parafuncional (Bruxismo). A dor foi caracterizada como sendo espontânea, contínua, localizada e severa, com a necessidade de uso de medicação analgésica. À inspeção verificou-se manchamento da coroa dentária e presença de selamento provisório da cavidade de acesso com material à base de óxido de zinco e eugenol. O dente apresentou sensibilidade aos testes de percussão horizontal e vertical, assim como, à palpação da mucosa vestibular por distal.

A radiografia final do tratamento endodôntico foi trazida pela paciente (Figura $1 \mathbf{a} \mathbf{e}$ b) onde foi possível constatar a presença de lesão periapical difusa e de uma reabsorção cervical envolvendo a coroa e a raiz na face distal. A fim de se completar o diagnóstico e avaliar o dano ao periodonto de sustentação, solicitou-se uma tomografia computadorizada cone-beam.

Figura 1 (a e b) - Radiografia final do tratamento endodôntico que foi trazida pela paciente, apresentando reabsorção cervical do tipo interna.

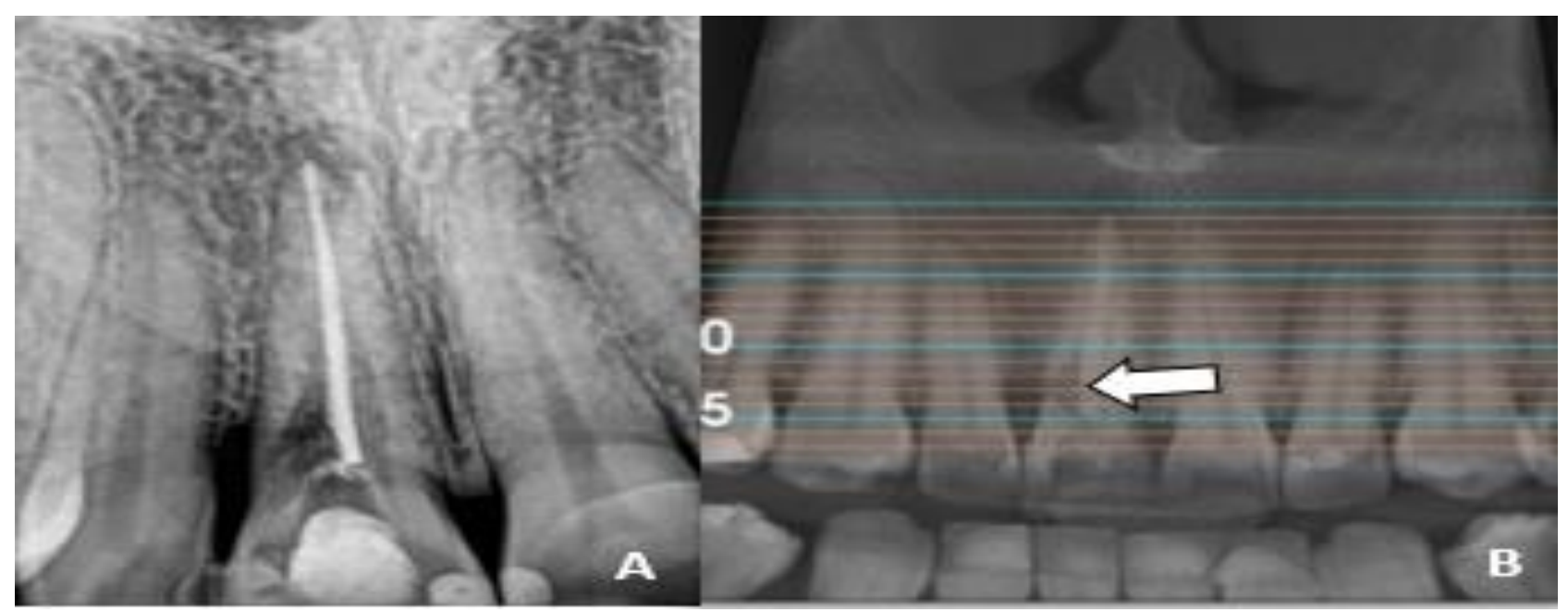

Fonte: Dados da pesquisa, 2018. 
Figura 2 - Cortes axiais da tomografia mostrando a reabsorção interna com comunicação com o periodonto lateral por distal.

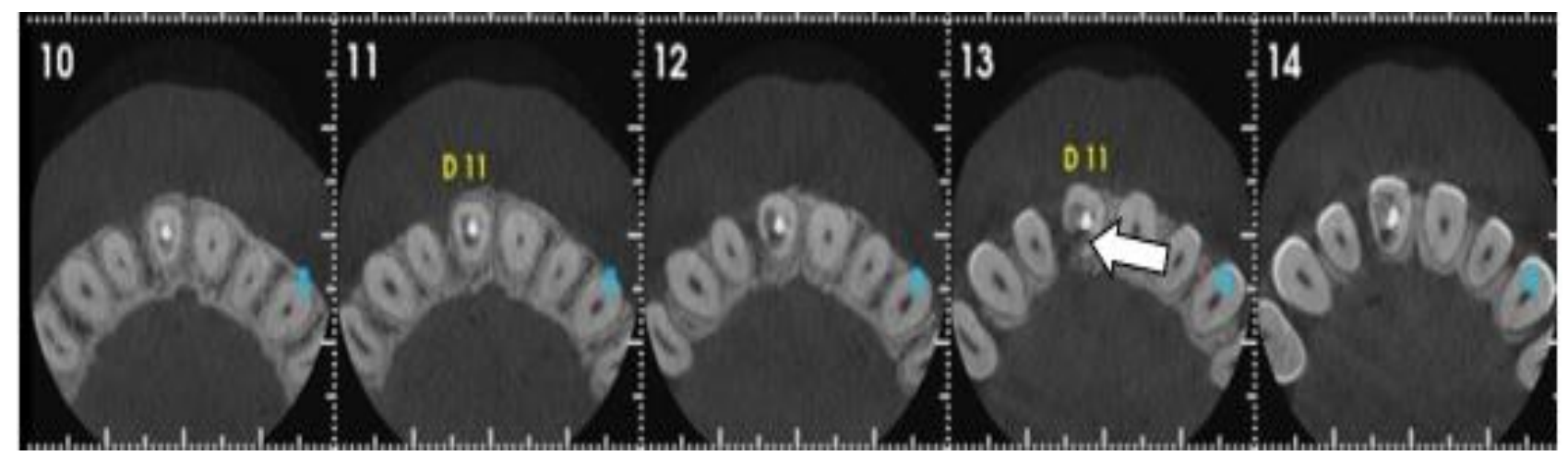

Fonte: Dados da pesquisa, 2018.

O resultado da tomografia confirmou a reabsorção cervical que foi classificada como do tipo interna com comunicação com o periodonto lateral por distal (Figuras 3 e 4 a). A proposta terapêutica foi o retratamento endodôntico seguido do selamento da cavidade oriunda da reabsorção com cimento reparador.

Na sessão posterior (uma semana de intervalo), procedeu-se à desobturação do conduto com auxílio de limas manuais sob o princípio coroa-ápice, até que uma lima tipo $\mathrm{K} \# 50$ alcançou o Comprimento de Trabalho de Exploração (Figura 4 a). Com o auxílio do localizador apical eletrônico - Gnatus $®$ - confirmou-se o Comprimento de Trabalho. Durante a desobturação ocorreu o extravasamento acidental de um fragmento de guta-percha para região periapical (Figura 3). O canal foi medicado com pasta Calen com PMCC® - SS White, a fim de que o mesmo permanecesse com a medicação por um intervalo de 15 dias.

Figura 3 - Extravasamento acidental de um fragmento de guta-percha para região periapical.

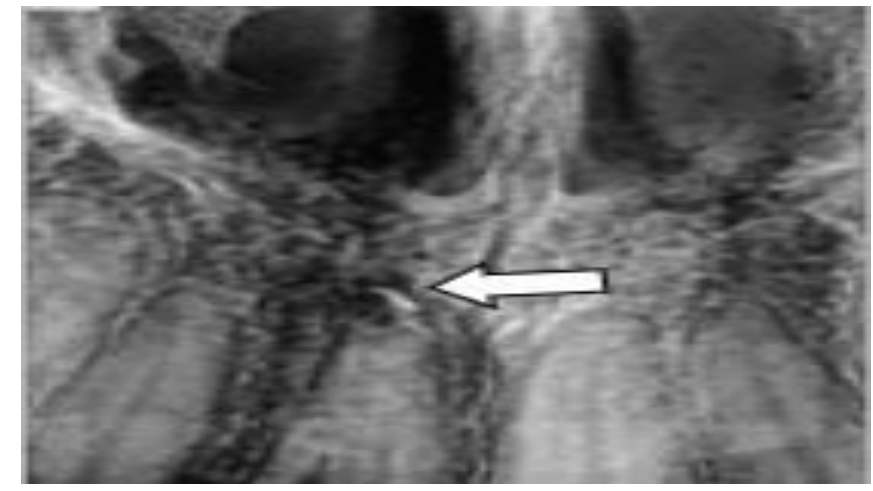

Fonte: Dados da pesquisa, 2018.

Na primeira sessão realizou-se anestesia terminal infiltrativa do nervo alveolar superior anterior pela técnica supraperiosteal com Lidocaína a 2\% 1: 100.000 Ul de epinefrina (Alphacaíne® - Nova DFL). Em seguida, sob isolamento absoluto (técnica do Isolamento em bloco) removeu-se o selamento provisório com uma ponta diamantada 1014, obtendo-se acesso ao material obturador e à cavidade da reabsorção. $\mathrm{O}$ objetivo desta sessão foi curetar e limpar ao máximo a cavidade de reabsorção para retirar o tecido de granulação presente. Para tanto, utilizou-se curetas do tipo longa e uma broca de Gates-Glidden No 3 com cuidado para não prejudicar a estrutura remanescente. Concomitantemente, procedeu-se irrigações frequentes com Hipoclorito de sódio a 2,5 \% (LAFEPE). Em seguida, preencheu-se a cavidade com pasta de Hidróxido de Cálcio com água destilada (Ultracal ${ }^{\circledR}$ - Ultradent) e a cavidade de acesso foi selada com cimento provisório (Coltosol® - Coltene). 
Estando a paciente assintomática e o canal livre de sangramento e exsudato, procedeu-se a obturação pela Técnica de compactação lateral e vertical, cortando-se a mesma ligeiramente abaixo do nível da cavidade da reabsorção. Em seguida, a cavidade de reabsorção foi preenchida com MTA Repair HP com auxílio de espátulas de inserção, calcadores de Paiva e compressão com mechas de algodão estéreis. O MTA foi protegido com uma camada de Coltosol e a cavidade de acesso foi selada com lonoseal $\AA^{\circledR}$ - Volco. $\mathrm{Na}$ radiografia final pode-se observar o completo selamento da cavidade de reabsorção (Figura 4 a e b).

Figura 4 - Prova do cone para realização da obturação pela técnica de compactação lateral e vertical (a), Radiografia final mostrando o completo selamento da cavidade de reabsorção (b).

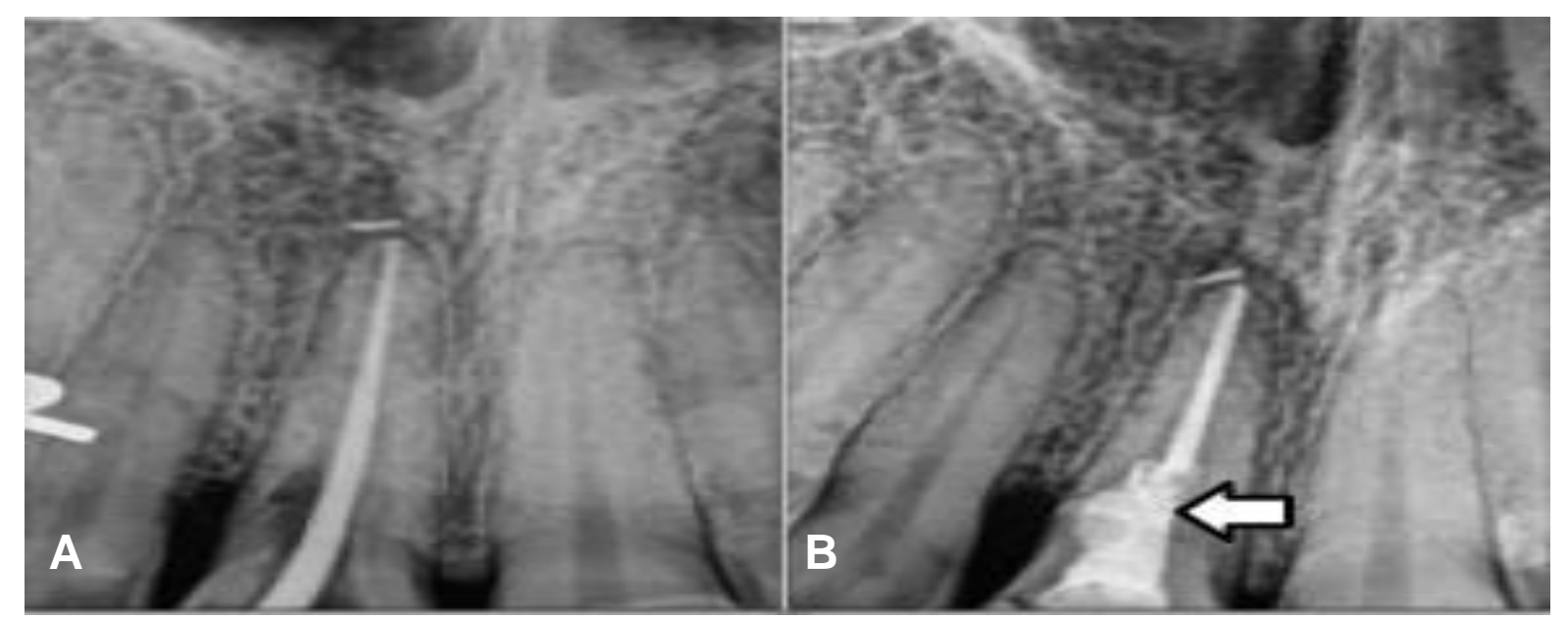

Fonte: Dados da pesquisa, 2018.

\section{DISCUSSÃO}

As reabsorções radiculares internas, em sua maioria não apresentam sintomatologia, porém durante o processo de reabsorção, pode ocorrer perfuração da parede dentinária e surgir assim sintomatologia dolorosa. Se a reabsorção estiver localizada na região coronária, o dente pode apresenta-se com uma coloração rosada, devido à presença de tecido de granulação (FERREIRA MM, LEITÃO J, CARRILHO EVP, 2007). No caso em questão, a sintomatologia foi caracterizada como sendo espontânea, contínua, localizada e severa, com a necessidade de uso de medicação analgésica, provavelmente devido ao não-tratamento da área comprometida pela reabsorção, somada a uma provável manutenção do quadro infeccioso que culminou na periodontite apical.

Os exames de imagem, são de extrema importância para a conclusão do diagnóstico, onde a tomografia computadorizada é muito utilizada na Endodontia para identificação e localização de reabsorções interna e externa, observação de anatomia radicular, detecção de fraturas radiculares verticais e planejamento cirúrgico (PATEL $S$ et al., 2010).

As reabsorções radiculares, radiograficamente aparecem como uma imagem radiolúcida, simétrica, ovóide ou arredondada, alterando o contorno original do canal radicular (MENDONÇA TA et al., 2012). Dessa forma, a fim de se completar o diagnóstico e avaliar o dano ao periodonto de sustentação, solicitou-se uma tomografia computadorizada cone-beam para o caso relatado, onde foi possível detectar a localização e a proporção da cavidade reabsortiva.

As reabsorções dentárias inflamatórias têm como princípio terapêutico a eliminação da causa. Quando o processo inflamatório e o estresse celular cessam na área em reabsorção, as unidades osteorremodeladoras e seus clastos sofrem uma desmobilização e saem da superfície radicular: os mediadores desaparecem. $O$ $\mathrm{pH}$ da região volta ao estado de neutralidade e novos cementoblastos são formados, recolonizando a superfície radicular em alguns dias. Forma-se, em seguida, novo cemento, com reinserção das fibras colágenas no meio da nova camada cementoblástica e assim a superfície radicular volta a ser biologicamente normal (CONSOLARO, 2011). 
O tratamento da Reabsorção Interna é feito na realização do preparo biomecânica do canal radicular, que se trata da remoção do tecido pulpar inflamado ou necrosado. Existem, no entanto, algumas complicações que poderão ser enfrentatas no momento do tratamento, como seja a dificuldade de os instrumentos conseguirem atingir e remover todo o tecido de granulação que se encontra na área de reabsorção (BRAMANTE CM, RAMOS CAS, 2001; BRAMANTE CM, 2003). No caso citado, o elemento dentário já estava tratado endodonticamente, fazendo-se necessário uma nova intervenção endodôntica devido à presença de sinais e sintomas, e, para remoção do tecido de granulação, foi necessário empregar curetas de haste longa e uma broca de Gates-Glidden \#3 a fim de regularizar as paredes dentinárias, favorecendo o posterior preenchimento com o cimento reparador.

Os materiais reparadores biocêramicos são bastante utilizados na endodontia, principalmente nos casos de prognósticos desfavoráveis. Suas aplicações clínicas vão desde um tratamento de proteção pulpar, reabsorções internas, perfurações, retro obturação em cirurgias endodônticas e até ao selamento apical emcasos de dentes com ápice aberto (LOVATO K E SEDGLEY C, 2011). Sendo o material de escolha para a obturação do canal radicular e da área de reabsorção o MTA HP, por ser um cimento reparador indutor de biomineralização.

Uma vez que a terapêutica da reabsorção interna e externa é diferente, é de suma importância a realização de um diagnóstico diferencial cuidadoso, onde a solicitação de exames, como tomografia computadorizada podem auxiliar aumentando a confiabilidade do diagnóstico. É válido ainda ressaltar que os cimentos biocêramicos possuem interação e resposta ao estímulo de tecidos vivos, tornando o prognóstico das reabsorções melhores. O tratamento realizado no caso relatado, resultou em uma resposta inicialmente favorável, porém, é preciso manter o controle clínico-radiográfico em consultas periódicas para proservação do caso.

\section{REFERÊNCIAS}

1. AZEVEDO KESQ. Cimento biocerâmico reparador: e suas propriedades na endodontia. Universidade Fernando Pessoa, Porto, 2017; 4 (2): 1-4.

2. BRAMANTE CM. Acidentes e Complicações no Tratamento Endodontico-Soluções Clínicas. Livraria Santos, 2003; 1(9): 184-197.

3. BRAMANTE CM, BERBERT A. Recursos radiográficos no diagnostico e no tratamento endodôntico, $2002 ; 3$ (1): 56 63.

4. BRAMANTE CM, RAMOS CAS. Endodontia Fundamentos Biológicos e Clínicos. $2^{2}$ ed. Santos livraria editora, 2001; cap.1(1): 32-36.

5. CAMARGO SEA. Principais características clínicas e radiográficas das reabsorções radiculares internas e externas. Revista de Odontologia da Universidade Cidade de São Paulo, 2008; 2 (20): 195-203.

6. FERREIRA MM, LEITÃO J, CARRILHO EVP. Reabsorção radicular interna. Revista Portuguesa de Estomatologia, Medicina Dentária e Cirurgia Maxilofacial, 2007; 2 (48): 121-126.

7. LEONARDO MR, LEAL JM. Endodontia: Tratamento de canais radiculares, São Paulo,1998; 3(20): 333-428.

8. LIMA SMF, REZENDE TMB. Benefícios de Exames Tomográficos na Endodontia: Revisão de Literatura. Oral Sci, $2011 ; 3(1): 26-31$.

9. LOVATO K, SEDGLEY C. Antibacterial Activity of EndoSequence Root Repair Material and ProRoot MTA® against Clinical Isolates of Enterococcus faecalis. Journal of Endodontics, 2011; 11(37): 1542-1546.

10. MENDONÇA TA. Uso da tomografia computadorizada por feixe cônico na endodontia. Revista Fluminense de Odontologia. Rio de Janeiro, 2012; 1 (37): 39-42.

11. MINUZZI ED. Reabsorção dentária externa: revisão de literatura e relato de caso clínico. Universidade Federal do Rio Grande do Sul. Faculdade de Odontologia. Curso de Especialização em Endodontia, 2017;.1-29.

12. NASCIMENTO GJF. Mecanismo, classificação e etiologia das reabsorções dentárias. Revista da Faculdade de Odontologia de Porto Alegre. Porto Alegre, 2006; 3 (47): 17-22.

13. NEVILLE BW. Patologia Oral e Maxilofacial. Trad. 3 eds., Rio de Janeiro: Elsevier, 2009; 64-69.

14. PATEL S, KANAGASINGAM S, MANNOCCI F. Cone beam computed tomography (CBCT) in endodontics. Dent Update, 2010; 37 (6): 373-9.

15. SOUZA NETO MD, PÉCORA JD, SAQUY PC. Traumatismo alvéolo-dentário. In: Estrela C, Figueiredo JAP. Endodontia: princípios biológicos e mecânicos. 1. ed. São Paulo: Artes Médicas, 2001; 761-85.

16. ZUOLO ML. Tratamento de perfuração dentária durante o retratamento endodôntico com o uso de um novo material baseado em MTA. Angelus. MTA REPAIR HP. 2016. 